\title{
Some Experimental Results on the Role of Speed and Accuracy of Reading in Psychometric Tests
}

\author{
Isabella Morlini, Giacomo Stella, and Maristella Scorza
}

\begin{abstract}
According to the Italian Parliament act (n. 170/2010) that recognizes dyslexia as a physical disturbance, of neurobiological origin, dyslexic children in primary school should be early recognized, in order to assess a targeted intervention within the school and to start a teaching that respects the difficulties in learning to read, to write, and to perform calculations. Screening procedures inside the primary schools aimed at detecting children with difficulties in reading are not so common in Italy as in other European countries. Nevertheless, screening procedures are of fundamental importance for guaranteeing an early detection of dyslexic children and reducing both the primary negative effects - on learning and the secondary negative effects — on the development of the personality — of this disturbance. In this study we analyze the validity, from a statistical point of view, of a screening procedure recently proposed in the psychometric literature (Stella et al., SPILLO Strumento per l'identificazione della lentezza nella lettura orale. Software per la verifica delle abilit di lettura dei bambini della scuola primaria (dalla prima alla quinta). Giunti Scuola, Florence, 2011). This procedure is very fast (it is exactly one minute long), simple, cheap and can be dispensed by teachers without psychometric experience. On the contrary, the currently used tests are much longer and must be provided by skilled teachers. These two major flaw prevent the widespread use of these tests. If the new procedure is found to
\end{abstract}

\footnotetext{
I. Morlini $(\bowtie)$

Department of Economics, University of Modena \& Reggio Emilia, Via Berengario 51, 41.121

Modena, Italy

e-mail: isabella.morlini@unimore.it

G. Stella • M. Scorza

Department of Social Sciences, University of Modena \& Reggio Emilia, Viale Allegri, 42.121

Reggio Emilia, Italy

e-mail: giacomo.stella@unimore.it; maristella.scorza@unimore.it
} 
be reliable, it can be provided to each student in primary school and it can also be repeated in time, in order to monitor the children difficulties. The validity of the procedure and the benchmark with two currently used tests are studied on the basis of the results of a survey on about 1,500 students attending primary school.

\section{Introduction}

The act of Parliament n. 170 (approved the 8th October 2010), on "the new statutory law for learning disorders affecting the scholastic population" states that dyslexia is a physical disturbance, of neurobiological origin, which makes it very difficult to learn to read, to write, and to perform calculations for intelligent children who do not have any other types of disorder. According to this act, teaching a dyslexic child in the school should respect his pace and learning methods and should include a system of assessment that takes into account his different performances. The early detection of dyslexic children in primary school becomes then of fundamental importance. Procedures for recognizing dyslexic children are based on reading performance. Indeed, even though Italians use a shallow orthography which facilitates reading, Paulesu et al. (2001) found that an Italian dyslexic reads better than a French and an English dyslexic, but he performs significantly worse than a non-dyslexic Italian reader. The aim of this paper is to study the validity, from a statistical perspective, of a screening procedure recently proposed in the psychometric literature (Stella et al. 2011). This procedure is very fast (it is exactly one minute long), simple and can be dispensed by teachers without psychometric experience. The reading tests currently used in Italy, in primary school, are much longer and must be dispensed by skilled teachers. Drawing from the results obtained by administering the screening procedure and, as a benchmark, two currently used reading tests, to about 1,500 students in primary school, in Italy, the purpose of this work is twofold:

- To study the empirical distribution of the variables measuring speed and accuracy of reading in the screening procedure and in the benchmark tests. This study allows us to discuss the validity of the threshold values used for classifying the student's performance as impaired or as not impaired.

- To study the validity of the screening procedure, through an explorative factor analysis and through the estimate of the internal consistency.

The paper is organized as follows. In Sect. 2 we briefly illustrate the screening procedure and the tests used as benchmarks. In Sects. 3 and 4 we report univariate and bivariate analyses of the variables measuring the reading performances, we analyze the empirical distribution of these variables and we discuss the choice of the threshold values currently used for classifying the student's performance. In Sect. 5 we analyze the validity of the screening procedure. Finally, in Sect. 6 we give some concluding remarks. 


\section{The Screening Procedure and the Benchmark Tests}

The new screening procedure for students attending primary school, in Italy, is called SPILLO. It is implemented within a software and thus the results of the screening are immediately available after the implementation. The student is asked to read a text for exactly $1 \mathrm{~min}$. The examiner clicks the word spelled wrongly on the screen and, after $1 \mathrm{~min}$, the software emits a sound that indicates the end of the procedure. The examiner clicks the last word read by the student and the software computes the number of words, the number of syllables read in a second, the number of errors and, eventually, the $z$-scores. Since the evaluation of reading ability should take into account both the single word reading process (which is an explicit and automatic process) and the lexical anticipation (which is a higher level process), in SPILLO the reading performances are assessed in a text rather than in a list of words or nonwords (words without a meaning). The text chosen is a story composed of 181 words. The variables measuring the reading performance are:

$Y_{1}$ : number of words read in a minute,

$Y_{2}$ : number of syllables per second read (in a minute).

$Y_{3}$ : number of wrong spelling in a minute.

The benchmark tests are two currently used "paper and pencil" tests. While the student reads, the examiner times the reading and makes a note of the mistakes. Then, the examiner classifies the student as an impaired reader or as a not impaired reader, on the basis of the normative threshold values. This procedure is very simple for experts but it may result rather difficult for examiners who lack a statistical background. Indeed, results are usually affected by many errors due to erroneous calculus or wrong interpretation of the results. In the first test the student is asked to read a list of words and in the second test a list of nonwords. These two lists have been introduced and studied by Sartori et al. (1995, 2007). In the two benchmark tests the variables measuring the reading performance are:

$X_{1}$ : time (in seconds) in reading the list of words,

$X_{2}$ : number of syllables per second read in the list of words,

$X_{3}$ : number of wrong spellings in reading the list of words,

$X_{4}$ : time (in seconds) in reading the list of nonwords,

$X_{5}$ : number of syllables per second read in the list of nonwords,

$X_{6}$ : number of wrong spellings in reading the list of nonwords.

The new screening procedure and the two benchmark tests have been provided to 1,469 students in elementary school, in the Lombardia and the Emilia Romagna regions (Northern Italy). The tests have been administered to students attending classes II-V in February and to students attending class I in May. Since Italian is a language with transparent or shallow orthography, where the letters of the alphabet, alone or in combination, are in most instances uniquely mapped to each of the speech sounds occurring in the language, at the end of the first school year students are in general able to read. The sample does not include foreign students. 
The composition of the sample is: 333 students attending class I, 384 students attending class II, 200 students attending class III, 276 students attending class IV, and 276 students attending class V.

\section{$3 \quad$ Reading Speed}

In Table 1 we list the values of some univariate statistics of the variables measuring the reading speed $\left(X_{1}, X_{2}, X_{4}, X_{5}, Y_{1}, Y_{2}\right)$. Figure 1 shows the empirical distributions of these variables through boxplots. Performances in reading speed improve from class I to class V: both the median and the mean values of $X_{1}$ and $X_{3}$ decrease while the mean and the median values of $X_{2}, X_{4}, Y_{1}$, and $Y_{2}$ increase. Variables measuring the number of words and the number of syllables read in a second have a similar pattern: the average values of $Y_{1}$ and $Y_{2}$ across the five classes have a behavior similar to the average values of $X_{2}$ and $X_{5}$. Dispersion, measured by the coefficient of variation, always decreases with the grade level. In the time of reading $\left(X_{1}\right.$ and $X_{4}$ ) and in $Y_{1}$ and $Y_{2}$, a drop of the coefficient of variation corresponding to class III is evident. This drop in not present in $X_{2}$ and $X_{5}$. The larger variability in classes I, II, and III may be explained by considering that many covariates (like the cultural level, the experiences in day nursery, etc...) have a great influence on the reading performances. From class III, in general, these covariates become irrelevant and the scholastic population becomes more homogeneous.

Variables measuring the speed of reading in the benchmark tests $\left(X_{1}, X_{2}, X_{4}\right.$, $\left.X_{5}\right)$ have a positive skew and present outlying values higher than $x_{0.75}-1.5\left(x_{0.75}-\right.$ $x_{0.25}$ ), in all classes. These characteristics are desirable for $X_{1}$ and $X_{4}$ that have a "positive direction of pathology" (impaired readers are children with high values in these variables), but not for $X_{2}$ and $X_{5}$ that have a "negative direction of pathology" (impaired readers are children having small values in these variables). $Y_{1}$ and $Y_{2}$ have a positive skew in classes I and II but a negative skew in classes II, IV, and V. In IV and $\mathrm{V}$ these variables have outlying values smaller than $x_{0.25}+1.5\left(x_{0.75}-x_{0.25}\right)$.

The currently used threshold for $X_{1}, X_{2}, X_{4}, X_{5}$ are based on the assumption of normality. The thresholds are used for classifying students as normal readers or impaired readers. They have been specified on the basis of the mean and the variance, assuming a normal distribution (Sartori et al. 1995, 2007). The thresholds have been obtained as $\mu+2 \sigma$ (for $X_{1}$ and $X_{4}$ ) and as $\mu-2 \sigma$ (for $X_{2}$ and $X_{5}$ ), where $\mu$ indicates the mean and $\sigma$ the standard deviation, considering that in a Gaussian distribution these values exclude about $2 \%$ of the population. The estimated values of $\mu$ and $\sigma$, reported in Sartori et al. (2007) and currently used as normative values in the tests, have been estimated for the classes II, III, IV, and V on a very small sample. Using the $T$-test for the means and the non-parametric test of Levene for the variances, they result significantly different $(\alpha=0.05)$ from the means and the variances obtained in our study and reported in Table 1. Moreover, with the three non-parametric tests of Shapiro-Wilk, Anderson-Darling, and Jarque-Bera, the null hypothesis of Gaussian distribution of the variables $X_{1}, X_{2}, X_{4}, X_{5}, Y_{1}, Y_{2}$, in each 
Table 1 Univariate statistics obtained in the sample for variables measuring the reading speed. Values in bold are the selected thresholds for the screening procedure

\begin{tabular}{|c|c|c|c|c|c|c|c|c|c|c|c|c|c|c|c|}
\hline & \multicolumn{5}{|l|}{$X_{1}$} & \multicolumn{5}{|l|}{$X_{4}$} & \multicolumn{5}{|l|}{$Y_{1}$} \\
\hline & I & II & III & IV & V & I & II & III & IV & V & I & II & III & IV & V \\
\hline $\mathrm{N}$. & 333 & 384 & 200 & 276 & 276 & 333 & 384 & 200 & 276 & 276 & 333 & 384 & 200 & 276 & 276 \\
\hline Min & 92 & 69 & 52 & 50 & 43 & 58 & 49 & 33 & 34 & 31 & 8 & 21 & 48 & 9 & 57 \\
\hline Max & 1148 & 595 & 289 & 348 & 206 & 542 & 311 & 210 & 234 & 118 & 127 & 157 & 164 & 180 & 180 \\
\hline$x_{0.05}$ & 145 & 96 & 70 & 59 & 54 & 89 & 68 & 52 & 49 & 38 & 16 & 34 & 64 & 78 & 96 \\
\hline$x_{0.25}$ & 232 & 135 & 90 & 73 & 64 & 120 & 89 & 69 & 59 & 50 & 30.0 & 54.0 & 77.0 & 108.0 & 127.0 \\
\hline$x_{0.50}$ & 314 & 177 & 110 & 89 & 74 & 151 & 110 & 84 & 72 & 59 & 41.0 & 66.0 & 97.0 & 125.0 & 146.0 \\
\hline$x_{0.75}$ & 424 & 234 & 146 & 108 & 90 & 204 & 137 & 98 & 86 & 73 & 53.0 & 81.0 & 115.3 & 140.0 & 164.0 \\
\hline$x_{0.95}$ & 723 & 381 & 209 & 153 & 121 & 342 & 206 & 157 & 109 & 97 & 72 & 113 & 138 & 170 & 180 \\
\hline Mean $(\bar{x})$ & 360 & 198 & 121 & 96 & 79 & 173 & 120 & 88 & 74 & 63 & 43.0 & 69.0 & 97.7 & 123.5 & 143.7 \\
\hline$S$ & 190.5 & 88.9 & 43.7 & 34.2 & 23.5 & 82.4 & 45.4 & 29.8 & 23.8 & 17.8 & 19.9 & 23.9 & 24.3 & 26.9 & 25.3 \\
\hline$S / \bar{x}$ & 0.53 & 0.45 & 0.36 & 0.36 & 0.30 & 0.48 & 0.38 & 0.34 & 0.32 & 0.28 & 0.46 & 0.35 & 0.25 & 0.22 & 0.18 \\
\hline \multirow[t]{3}{*}{ Skewness } & 1.66 & 1.49 & 1.30 & 2.53 & 1.84 & 1.76 & 1.64 & 1.45 & 2.23 & 0.61 & 1.21 & 0.64 & -0.23 & -0.42 & -0.76 \\
\hline & \multicolumn{5}{|l|}{$X_{2}$} & \multicolumn{5}{|c|}{$X_{5}$} & \multicolumn{5}{|c|}{$Y_{2}$} \\
\hline & $\mathrm{I}$ & II & III & IV & V & $\mathrm{I}$ & II & III & IV & $\mathrm{V}$ & $\mathrm{I}$ & II & III & IV & $\mathrm{V}$ \\
\hline Min & 0.24 & 0.47 & 0.97 & 0.80 & 1.36 & 0.23 & 0.41 & 0.60 & 0.54 & 1.07 & 0.30 & 0.78 & 1.77 & 0.32 & 2.07 \\
\hline Max & 3.04 & 4.06 & 5.38 & 5.60 & 6.51 & 2.17 & 2.57 & 3.82 & 3.71 & 4.06 & 4.23 & 5.25 & 5.53 & 5.98 & 5.98 \\
\hline$x_{0.05}$ & 0.39 & 0.73 & 1.34 & 1.83 & 2.31 & 0.37 & 0.61 & 0.80 & 1.16 & 1.30 & 0.58 & 1.27 & 2.30 & 2.80 & 3.32 \\
\hline$x_{0.25}$ & 0.66 & 1.20 & 1.92 & 2.59 & 3.11 & 0.62 & 0.92 & 1.29 & 1.47 & 1.73 & 1.10 & 1.97 & 2.77 & 3.63 & 4.23 \\
\hline$x_{0.50}$ & 0.89 & 1.58 & 2.56 & 3.16 & 3.78 & 0.83 & 1.15 & 1.51 & 1.76 & 2.14 & 1.48 & 2.38 & 3.33 & 4.18 & 4.87 \\
\hline$x_{0.75}$ & 1.21 & 2.08 & 3.12 & 3.84 & 4.38 & 1.05 & 1.42 & 1.83 & 2.14 & 2.52 & 1.95 & 2.90 & 3.86 & 4.65 & 5.53 \\
\hline$x_{0.95}$ & 1.93 & 2.92 & 4.00 & 4.75 & 5.19 & 1.42 & 1.85 & 2.43 & 2.82 & 3.32 & 2.65 & 3.82 & 4.55 & 5.70 & 5.98 \\
\hline Mean $(\bar{x})$ & 0.98 & 1.67 & 2.59 & 3.20 & 3.77 & 0.86 & 1.18 & 1.59 & 1.85 & 2.17 & 1.56 & 2.46 & 3.36 & 4.16 & 4.82 \\
\hline$S$ & 0.48 & 0.66 & 0.81 & 0.89 & 0.92 & 0.33 & 0.37 & 0.49 & 0.53 & 0.62 & 0.68 & 0.77 & 0.73 & 0.81 & 0.79 \\
\hline$S / \bar{x}$ & 0.49 & 0.40 & 0.31 & 0.28 & 0.24 & 0.38 & 0.32 & 0.31 & 0.29 & 0.29 & 0.44 & 0.31 & 0.22 & 0.19 & 0.16 \\
\hline Skewness & 1.48 & 0.74 & 0.34 & 0.16 & 0.03 & 0.54 & 0.45 & 0.83 & 0.72 & 0.69 & 1.06 & 0.45 & -0.09 & -0.34 & -0.69 \\
\hline
\end{tabular}

class, is rejected, even for $\alpha=0.001$. The normative thresholds, in our sample, lead to the following percentages of students classified as impaired readers:

\begin{tabular}{lllll}
\hline & $X_{1}(\%)$ & $X_{2}(\%)$ & $X_{4}(\%)$ & $X_{5}(\%)$ \\
\hline Class II & 7.8 & 0.5 & 5.7 & 0.0 \\
\hline Class III & 3.0 & 0.0 & 4.5 & 0.5 \\
\hline Class IV & 2.5 & 1.1 & 1.4 & 0.7 \\
\hline Class V & 2.2 & 0.7 & 1.1 & 0.0 \\
\hline
\end{tabular}

These percentages vary greatly not only across the classes but also across the variables. In class II, for example, 30 students are classified as impaired readers with $X_{1}$ and only 2 with $X_{2}$. Quite all the percentages are far from the expected value, equal to the estimated percentage of dyslexic students in the Italian population (about $4 \%$ ). Due to the non-normality of the variables and the presence of outliers, thresholds for $Y_{1}$ and $Y_{2}$ have been set equal to the percentile $x_{0.5}$ obtained in our 


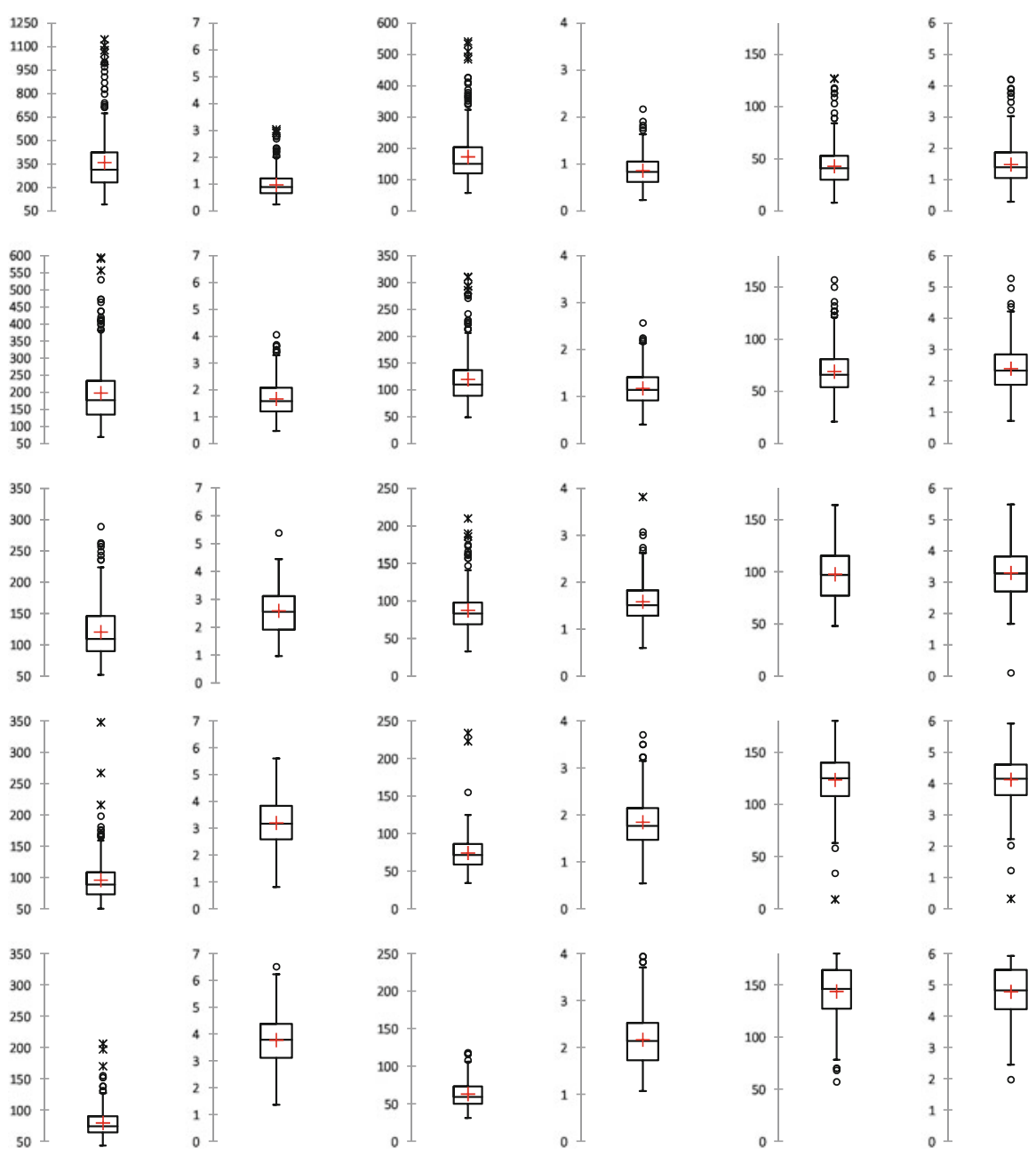

Fig. 1 Boxplots of the following variables (from the left): $X_{1}, X_{2}, X_{4}, X_{5}, Y_{1}, Y_{2}$. The first line reports boxplots in class I, the second line in class II, the third line in class III, the fourth line in class IV, and the fifth line in class $\mathrm{V}$

sample (the values are reported in bold in Table 1) for discriminating a percentage of people slightly higher than the expected percentage. As a matter of fact, the procedure is not intended as a diagnostic test for learning disorders but as a screening for detecting students with heavy difficulties in reading. The causes of these difficulties are to be defined by subsequent more detailed analyses. Future studies are necessary to explore the percentages of students classified as impaired readers with these thresholds in a new sample of students.

To investigate the validity of the screening procedure, we may analyze the pairwise correlations between the variables $Y_{1}$ and $Y_{3}$ and the variables used in 
Table 2 Correlation matrices for variables measuring reading speed in classes I and IV

\begin{tabular}{|c|c|c|c|c|c|c|c|c|c|c|c|c|}
\hline \multicolumn{6}{|c|}{ Class I } & \multicolumn{7}{|c|}{ Class IV } \\
\hline $\mathrm{X}_{1}$ & $\mathrm{X}_{2}$ & $\mathrm{X}_{4}$ & $\mathrm{X}_{5}$ & $\mathrm{Y}_{1}$ & $\mathrm{Y}_{2}$ & & $\mathrm{X}_{1}$ & $\mathrm{X}_{2}$ & $\mathrm{X}_{4}$ & $\mathrm{X}_{5}$ & $\mathrm{Y}_{1}$ & $\mathrm{Y}_{2}$ \\
\hline$X_{1} \quad 1$ & -0.79 & 0.91 & -0.81 & -0.72 & -0.73 & $X_{1}$ & 1 & -0.89 & 0.84 & $4-0.71$ & $1-0.76$ & -0.75 \\
\hline$X_{2}-0.79$ & 1 & -0.72 & 0.9 & 0.91 & 0.91 & $X_{2}$ & -0.89 & 1 & -0.77 & $\begin{array}{ll}7 & 0.83\end{array}$ & $\begin{array}{ll}3 & 0.77\end{array}$ & 0.76 \\
\hline $\begin{array}{ll}X_{4} & 0.91\end{array}$ & -0.72 & 1 & -0.86 & -0.66 & -0.66 & $X_{4}$ & 0.84 & -0.77 & 1 & -0.88 & $8-0.61$ & -0.61 \\
\hline$X_{5}-0.81$ & 0.9 & -0.86 & 1 & 0.82 & 0.82 & $X_{5}$ & -0.71 & 0.83 & -0.88 & $8 \quad 1$ & 0.61 & 0.6 \\
\hline$Y_{1}-0.72$ & 0.91 & -0.66 & 0.82 & 1 & 1 & $Y_{1}$ & -0.76 & 0.77 & -0.61 & 0.61 & 1 & 1 \\
\hline$Y_{2}-0.73$ & 0.91 & -0.66 & 0.82 & 1 & 1 & $Y_{2}$ & -0.75 & 0.76 & -0.61 & 0.6 & 1 & 1 \\
\hline
\end{tabular}

the benchmark tests. The correlation matrices for class I and IV are reported in Table 2. The matrices obtained in class II, III, and V look very similar. The pairwise correlations are all significantly different from zero $(\alpha=0.01)$. Even though the transformation from $X_{1}$ to $X_{2}$ and from $X_{3}$ to $X_{4}$ is not a linear one, the values of these pairs of variables are highly correlated. The transformation from $Y_{1}$ to $Y_{2}$ is not a perfect linear transformation (since the words have different numbers of syllables) but the correlation is nevertheless equal to 1 . The fact that $Y_{1}$ to $Y_{2}$ are highly correlated with $X_{1}, X_{2}, X_{4}$, and $X_{5}$ gives evidence that all these variables are a measure of the same aspect of the phenomenon dyslexia.

\section{$4 \quad$ Reading Accuracy}

In Table 3 we list the values of some univariate statistics in the variables measuring the accuracy of reading $\left(X_{3}, X_{6}, Y_{3}\right)$. Figure 2 shows the empirical distributions of these variables through boxplots. As well as the variables measuring the speed of reading, $X_{3}, X_{6}$, and $Y_{3}$ have an empirical distribution which is asymmetric and far from the Gaussian. While the mean and the median values of $X_{3}$ and $X_{6}$ have a decreasing pattern, from class I to class $\mathrm{V}$, the mean and the median values of $Y_{3}$ are roughly constant across classes. This different pattern is due to the fact that the time in the screening procedure is always equal to $1 \mathrm{~min}$, while it depends on the ability of the student in the benchmark tests. In the screening, if one student increases the performance from one class to the subsequent class, he increases the reading speed without penalizing the reading accuracy. Outliers are all in the "direction of pathology" and this is a desirable property. The normative threshold values for $X_{3}$ and $X_{6}$ are the 95th percentiles obtained in the study of Sartori et al. (2007). These values are similar to $x_{0.95}$ obtained in our sample (reported in Table 3 ). The percentages of students classified as impaired readers with the currently used thresholds are as follows: class II: $2.9 \%\left(X_{3}\right), 4.4 \%\left(X_{6}\right)$, class III: $1.5 \%\left(X_{3}\right)$, $4.0 \%\left(X_{6}\right)$, class IV: $4.3 \%\left(X_{3}\right), 9.4 \%\left(X_{2}\right)$, class V: $3.6 \%\left(X_{1}\right), 4.0 \%\left(X_{2}\right)$. These percentages are in general in agreement with the expected value. In the screening, the threshold valued in $Y_{3}$ is the value $x_{0.95}$ obtained in our sample. While $X_{3}$ and $X_{6}$ are highly correlated, $Y_{3}$ is only slightly positive correlated with $X_{3}$ or 
Table 3 Univariate statistics obtained in the sample for variables measuring the reading accuracy. Bold values are the selected thresholds for the screening procedure

\begin{tabular}{llllllllllllllllll}
\hline & $X_{3}$ & & \multicolumn{1}{c}{$X_{6}$} & & & & & $Y_{3}$ & & & \\
& I & II & III & IV & V & I & II & III & IV & V & I & II & III & IV & V \\
\hline Min & 0 & 0 & 0 & 0 & 0 & 0 & 0 & 0 & 0 & 0 & 0 & 0 & 0 & 0 & 0 \\
\hline Max & 84 & 55 & 18 & 19 & 22 & 38 & 31 & 20 & 24 & 27 & 9 & 10 & 6 & 11 & 11 \\
\hline$x_{0.25}$ & 5.0 & 4.0 & 2.0 & 1.0 & 1.0 & 4.0 & 5.0 & 3.0 & 3.0 & 2.0 & 0.00 & 0.50 & 0.50 & 0.88 & 0.50 \\
\hline$x_{0.50}$ & 10.0 & 6.0 & 4.0 & 3.0 & 2.0 & 8.0 & 7.0 & 6.0 & 5.0 & 4.0 & 1.00 & 1.50 & 1.50 & 1.00 & 1.00 \\
\hline$x_{0.75}$ & 17.0 & 10.3 & 6.0 & 5.0 & 4.0 & 13.0 & 10.0 & 8.0 & 8.0 & 6.0 & 2.00 & 2.50 & 2.00 & 2.50 & 2.00 \\
\hline$x_{0.95}$ & 38 & 19 & 11 & 10 & 9 & 24 & 17 & 14 & 14 & 12 & $\mathbf{4 . 0}$ & $\mathbf{5 . 0}$ & $\mathbf{4 . 0}$ & $\mathbf{4 . 0}$ & $\mathbf{4 . 0}$ \\
\hline Mean $(\bar{x})$ & 13.57 & 7.96 & 4.48 & 3.61 & 2.8 & 9.83 & 8.16 & 6.29 & 5.7 & 4.8 & 1.30 & 1.76 & 1.41 & 1.69 & 1.48 \\
\hline$S$ & 12.34 & 6.78 & 3.56 & 3.34 & 3.1 & 7.20 & 5.13 & 4.02 & 4.4 & 4.1 & 1.42 & 1.51 & 1.18 & 1.52 & 1.53 \\
\hline$S / \bar{x}$ & 0.91 & 0.85 & 0.79 & 0.93 & 1.12 & 0.73 & 0.63 & 0.64 & 0.76 & 0.84 & 1.09 & 0.86 & 0.84 & 0.90 & 1.03 \\
\hline Skewness & 2.07 & 2.62 & 1.29 & 1.63 & 2.55 & 1.29 & 1.18 & 1.06 & 1.32 & 1.92 & 1.80 & 1.35 & 0.97 & 1.81 & 2.29 \\
\hline
\end{tabular}
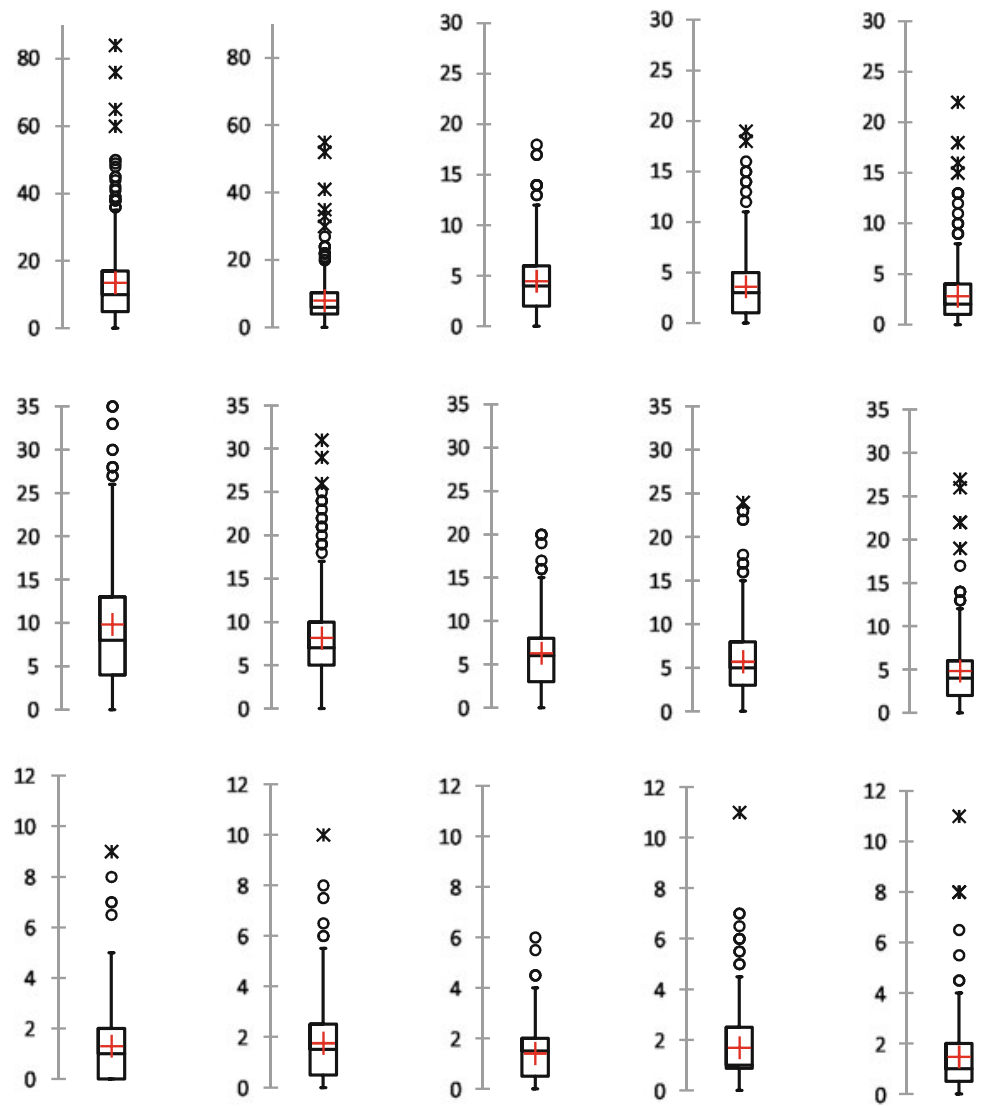

Fig. 2 Boxplots of $X_{3}$ (first line), $X_{6}$ (second line), and $Y_{3}$ (third line). Class I is in the first column, class II in the second, class III in the third, class IV in the fourth, and class V in the fifth column 
Table 4 Correlation matrices for variables measuring the reading accuracy

\begin{tabular}{|c|c|c|c|c|c|c|c|c|c|c|c|c|c|c|c|}
\hline \multicolumn{4}{|c|}{ Class I } & \multicolumn{3}{|c|}{ Class II } & \multicolumn{3}{|c|}{ Class III } & \multicolumn{3}{|c|}{ Class IV } & \multicolumn{3}{|c|}{ Class V } \\
\hline & $X_{3}$ & $X_{6}$ & $Y_{3}$ & $X_{3}$ & $X_{6}$ & $Y_{3}$ & $X_{3}$ & $X_{6}$ & $Y_{3}$ & $X_{3}$ & $X_{6}$ & $Y_{3}$ & $X_{3}$ & $X_{6}$ & $Y_{3}$ \\
\hline$X_{3}$ & 1 & 0.8 & 0.37 & 1 & 0.69 & 0.43 & 1 & 0.68 & 0.31 & 1 & 0.61 & 0.34 & 1 & 0.61 & 0.35 \\
\hline$X_{6}$ & 0.8 & 1 & 0.38 & 0.69 & 1 & 0.39 & 0.68 & 1 & 0.33 & 0.61 & 1 & 0.28 & 0.61 & 1 & 0.3 \\
\hline$Y_{3}$ & 0.37 & 0.38 & 1 & 0.43 & 0.39 & 1 & 0.31 & 0.33 & 1 & 0.34 & 0.28 & 1 & 0.35 & 0.3 & 1 \\
\hline
\end{tabular}

with $X_{6}$ (Table 4). This is due to the fact that the time is fixed in the screening. As explained previously, the number of mistakes has a different pattern from the number of mistakes in a reading test where the time depends on the ability of the subject.

\section{$5 \quad$ Internal Consistency}

An explorative factor analysis has been performed in order to investigate the multivariate relationships among variables used in the benchmark tests and in the screening procedure. The analysis, performed on the correlation matrix (reached with the values of all variables in all classes) shows the presence of two main latent orthogonal factors both with the principal component (PC) and with the common factors (CF) method. The first factor is highly correlated with variables measuring speed and, to a less degree, with $X_{3}$ and $X_{6}$. With the PC method, the eigenvalue of this factor is equal to 6 and the percentage of explained variance is $66.7 \%$. With the FC method, the eigenvalue is 5.83 and the percentage of variance $64.8 \%$. The second factor is highly correlated with $Y_{3}$ and, to a less degree, with the other variables measuring accuracy $\left(X_{3}\right.$ and $X_{6}$ ). With the PC method, the eigenvalue of this factor is 1.28 and the percentage of explained variance is $14.3 \%$. With the CF method, the eigenvalue is 0.86 and the percentage of variance $9.6 \%$. Figure 3 and Table 5 summarize results obtained with the PC method. Drawing from these results, with the belief that speed and accuracy of reading are two different aspects of dyslexia, we estimate the degree to which the set of variables $X_{1}, X_{2}, X_{4}, X_{5}$, $Y_{1}, Y_{2}$ measures a single unidimensional latent construct (the speed of reading) and the set of variables $\left(X_{3}, X_{6}, Y_{3}\right)$ measures an other unidimensional latent construct (the accuracy of reading). We estimate the internal consistency of each set of variables by means of the coefficient $\omega$ (McDonald 1999; Zinbarg et al. 2005), considering the correlation matrix. For the variables regarding speed, $\omega=0.86$. For the variables regarding accuracy, $\omega=0.64$. Since these variables have all positive pairwise correlations we may also calculate the $\alpha$ coefficient of Cronbach (1951) and the $\rho^{*}$ coefficient of Brown (1910) used in the psychometric literature. We obtain $\alpha=0.85$ and $\rho^{*}=0.70$. Regarding the speed of reading, if we select variables having positive pairwise correlations (namely, $X_{2}, X_{5}, Y_{1}, Y_{2}$ ), $\omega$ is 0.94 and both the $\alpha$ coefficient and the $\rho^{*}$ coefficient are 0.98 . Even though, in each set, the standardized variables are not $\tau$-equivalent and $\alpha$ gives an overestimation 


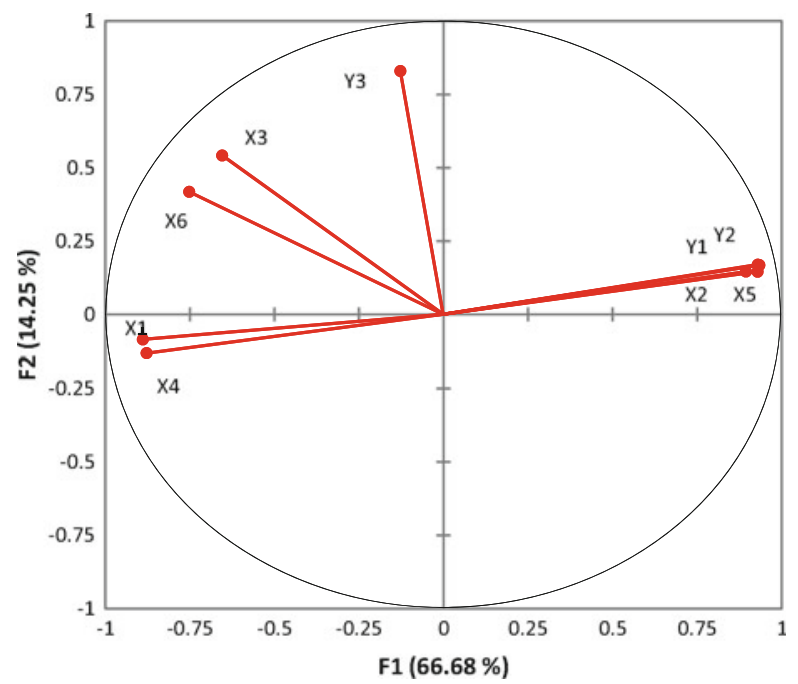

Fig. 3 Biplot resulting from factor analysis applied to the correlation matrix. The factors are extracted with the principal component method and are unrotated

Table 5 Correlations between factors and variables

\begin{tabular}{llllllllll}
\hline Factor & $X_{1}$ & $X_{2}$ & \multicolumn{1}{c}{$X_{3}$} & $X_{4}$ & $X_{5}$ & \multicolumn{1}{c}{$X_{6}$} & $Y_{1}$ & $Y_{2}$ & \multicolumn{1}{c}{$Y_{3}$} \\
\hline$F_{1}$ & -0.89 & 0.93 & -0.65 & -0.88 & 0.90 & -0.75 & 0.93 & 0.94 & -0.13 \\
\hline$F_{2}$ & -0.09 & 0.15 & 0.54 & -0.13 & 0.15 & 0.42 & 0.17 & 0.17 & 0.83 \\
\hline
\end{tabular}

The factors are extracted with the principal component method and are unrotated

of the internal consistency, all indexes show high inter-correlation among variables belonging to each set.

Since the screening procedure measures two latent constructs of the dyslexia phenomenon, to evaluate its reliability, we may consider the percentage of variance explained by the first two factors. With both the PC and the PF method of extraction, this percentage is $99.97 \%$ and indicates a very high reliability.

\section{Conclusions}

In this paper we have studied the validity, from a statistical perspective, of a new screening procedure proposed in the psychometric literature for the early detection of dyslexia in primary school. On the basis of the results obtained in a sample of 1,469 students we have shown that this screening procedure is able to measure speed and accuracy of reading as well as the currently used tests. The analysis of the empirical distribution of the variables measuring the reading performance in the tests has shown that the normative thresholds, used for classifying a student as a normal reader or as an impaired reader, do not seem to be trustworthy. Indeed, in 
our study the variables are found to be far from the normal distribution whereas the assumption of normality has been used to defined these thresholds. Moreover, the means and the variances that we have obtained in our sample are statistically different from the means and the variances used as normative values.

\section{References}

Brown, W.: Some experimental results in the correlation of mental abilities. Br. J. Psychol. 3, 296$322(1910)$

Cronbach, L.J.: Coefficient alpha and the internal structure of tests. Psychometrika 16(3), 297-334 (1951)

McDonald, R.P.: Test Theory: A Unified Treatment, pp. 90-103. Erlbaum, Mahwah (1999)

Paulesu, E., Demonet, J.F., Fazio, F., McCrory, E., Chanoine, V., Brunswick, N., Cappa, S.F., Cossu, G., Habib, M., Frith, C.D., Frith, U.: Dyslexia: cultural diversity and biological unity. Sciences 291, 2165-2167 (2001)

Sartori, G., Job, R., Tressoldi, P.E.: DDE Batteria per la valutazione della dislessia e della disortografia evolutiva. Giunti O.S., Firenze (1995)

Sartori, G., Job, R., Tressoldi, P.E.: DDE-2 Batteria per la valutazione della dislessia e della disortografia evolutiva - 2. Giunti O.S., Firenze (2007)

Stella, G., Scorza, M., Morlini, I.: SPILLO Strumento per l'identificazione della lentezza nella lettura orale. Software per la verifica delle abilit di lettura dei bambini della scuola primaria (dalla prima alla quinta). Giunti Scuola, Florence (2011)

Zinbarg, R., Revelle, W., Yovel, I., Li, W.: Cronbach's, Revelle's, and McDonald's: their relations with each other and two alternative conceptualizations of reliability. Psychometrika 70, 123-133 (2005) 